\title{
Exploring the concept of physical education curriculum redesign
}

\author{
Keejoon Yoon ${ }^{1}$ \& Ji-Young Seo $^{2 *}$ \\ ${ }^{1}$ Incheon National University \& ${ }^{2}$ Korean Institute for Curriculum and Evaluation
}

\begin{abstract}
[Purpose] The purpose of this study was to explore the concept of physical education redesign. [Methods] Studies on curriculum redesign, physical education curriculum redesign and the revised 2015 physical education curriculum were collected and analysed. [Results] First, three different types of curriculum redesign were discussed. Second, there was no clear concept of physical education redesign. Third, both 'learning content area' and 'standard achievement' were related to the physical education curriculum redesign. [Conclusions] Two different aspects of physical education curriculum redesign emerged. Establishing the clear concept of physical education curriculum redesign and presenting the concept in the next national physical education curriculum were suggested.
\end{abstract}

Key words: curriculum redesign, physical education curriculum, the 2015 revised physical education curriculum

\section{서 론}

A교사: 건도경표안(건강, 도전, 경쟁, 표현, 안전) 꼭 다 해 야 돼요? 안전은 아무리 생각해도 독립된 내용으로 짜는 것이 어렵네요. 만들어도 너무 억지스러운 것 같고. 그렇다고 안 할 수는 없고.

B교사: 내용체계표에 나오니까 다 해야 되는 거 아니에요?

C교사: 지난번 연수에서 들었는데 교육과정을 재구성 할 수 있다던데요? 그러면 다 안 해도 되는 거 아니에요?

A교사: 그래도 다섯 가지 영역은 모두 가르치는 거 아닌가 요? 그렇게 마음대로 바꿀 수 있나요?

논문 투고일 : 2020. 08. 24.

논문 수정일 : 2020. 10. 05.

게재 확정일 : 2020. 10. 23.

* 교신저자 : 서지영 (lovekid@kice.re.kr).

* 이 논문은 2019년도 인천대학교의 지원(2019-0464)을 받아 수행된 연구임.
같은 학교에서 근무하는 체육교사들이 연간 체육과 교 육과정을 개발하는 과정에서 논의할 수 있을 법한 내용을 구성한 것이다. C교사는 '교육과정 (문서)을 그대로 따르 지 않고 학교 상황에 맞도록 변형할 수 있다라는 내용을 직무연수에서 들은 경험이 있다. 그럼에도 국가수준 교육 과정 문서에서 제시하고 있는 내용 중에서 '무엇을', 그리 고 '어떻게' 재구성 할 수 있는 지에 대해서는 모른다. 세 교사 모두 뚜렷한 답을 내놓기 어렵다. 어렴풋이 단위학 교마다 구비하고 있는 시설 및 용·기구가 다르고 학급의 학생 수가 다를 수밖에 없기 때문에 일정 부분 변화가 필 요하다는 정도만 인식하고 있다. 이는 최초 제6차 교육과 정에서 '교수/학습 내용의 선택' 및 ‘수업 시간 운영' 영역 에서 교사에게 일정 수준의 자율성을 부여한 교육과정 역 사에서 기인한 것으로 분석된다(Ministry of Education, 1992, cited in Kim \& Lee, 1999, p. 36). 2015 개정 교육과정 총론에서도 '복식 학급을 편성·운영하는 경우에 는 교육내용의 학년별 순서를 조정하거나 공통 주제를 중 심으로 교재를 재구성하여 활용할 수 있다라고 제시하고 있다(Ministry of Education, 2015a, p. 10). 
그래서일까. 교육과정 재구성은 교사 및 교육과정 전 문가들에게 꽤 친숙한 개념으로 자리 잡았다. 관련 연구 역시 지속적으로 진행되었다. 지금까지 교육과정 재구성 은 수업을 설계하고 및 교과서의 내용을 새롭게 조직하는 방법(Sung \& Lee, 2009)에서부터 교사의 교수/학습 개 발까지도 포함하는 입장(Hur, 2011)까지 다소 폭 넓게 정의되고 있다. 국가수준 교육과정에서 제시하고 있는 내 용을 근무하고 있는 학교의 상황에 맞게 탄력적으로 변형 및 운영하는 것은 급변하는 교육 환경 변화에 교사가 반 드시 갖추어야 할 역량으로 이해되기도 한다. 최근 교사 의 '교육과정 문해력(curriculum literacy)'의 개념을 탐 색하는 연구가 관심 받고 있는 것도 같은 맥락에서 이해 할 수 있다(Park, Jeong \& Kim, 2017; Jeong, 2012). '교사 상상력'(Hur, 2011) 또는 '교육과정 가능성' (Kim \& Jung, 2013)과 같은 유사한 개념 역시 활발하게 연구 되고 있다.

문제는 교육과정 재구성이라는 개념이 생각보다 명확 하지 않다는 것이다(Kim, 2018). '교육과정 재구성의 개 념적 애매성과 비판' $(\mathrm{Seo}, 2011)$ 또는 '교육과정 재구성 논쟁' $(\mathrm{Seo}, 2016)$ 과 같은 다소 과격한 제목의 논문이 발 표되고 있는 점을 고려하면, 교육과정을 전공하는 학자들 에게도 교육과정 재구성은 명확한 개념으로서의 실체를 제공하지 못하는 형국이다. 명확한 개념이 제기되지 못한 상태는 논의의 진전 및 현장 개선을 어렵게 만든다. 앞서 예문의 $\mathrm{A}, \mathrm{B}, \mathrm{C}$ 교사가 생각하는 단위학교 연간 체육과 교육과정 개발과 관련된 의견들이 학계에서도 반복되는 것은 적절하지 않다. 교육과정 대강화(slimming)가 지속 적으로 논의되고 있는 학계의 분위기 또한 교육과정 재구 성의 개념 정립의 필요성을 뒷받침한다(Lee, 2019).

체육과 교육과정 재구성은 적어도 20년 이상 된 오래 된 개념이다. '선생님께서는 교육과정을 재구성하여 운영 하십니까?라는 설문 문항에 $50 \%$ 가 넘는 초등학교 교사 들이 ‘네’라고 응답한 연구 결과를 고려하면 (Kim \& Lee, 1999), 스포츠교육학자와 체육교사에게 교육과정 재구 성은 다소 친숙한 개념이라고 할 수 있다. 그러나 Park $\&$ Park (2006, p. 309)이 '교육과정 운영에서 핵심이라 고 할 수 있는 교사들의 교육과정 재구성에 대한 보다 심 도 깊은 연구는 미흡했다고 볼 수 있다'고 주장한 것과 같 이, 체육과 교육과정 재구성을 학술적으로 살펴보고자 하
는 노력은 부족했다.

그동안 수행된 체육과 교육과정 재구성 관련 연구1)는 다음과 같은 유형으로 구분할 수 있다. 첫째, 체육교사의 교육과정 재구성 실천 과정의 의미를 탐색하는 것이다. Kim et al. (2012)은 중학교 체육교사 4명의 체육과 교 육과정 재구성 경험을 '계획', '실천', '의미'로 구분하여 해 석하였다. Zae \& Youn (2015)은 초임 체육교사가 교육 과정 재구성을 경험하는 과정에서 겪는 어려움, 극복 전 략, 효과를 제시하였다. 둘째, 체육교사의 교육과정 실태 및 방법을 살펴보는 것이다. Kim \& Lee (1999)는 초등 학교 교사의 체육과 교육과정 재구성 경험 유무, 성별 및 경력에 따른 차이, 구체적인 재구성 유형 등을 탐색하였 다. Park \& Park (2006)은 초등학교 교사들이 교육과 정 재구성이 필요하다고 생각하는 이유, 재구성에 영향을 미치는 요인(시설, 용·기구, 학생 흥미), 효과적인 교육과 정 재구성에 요청되는 조건 등을 제시하였다. 셋째, 교육 과정 재구성 활용 방안을 제시하는 것이다. Chang \& Ko (2008)은 2007 개정 체육과 교육과정에서 처음으로 제 시한 신체활동 가치 중심 교수·학습 방법에 주목하여 재 구성의 의미를 해석하였다. 운동 기능 숙달 중심 체육교 육에서 신체활동 가치 중심 체육교육으로 전환하는 데 교 육과정 재구성이라는 아이디어를 차용한 것이다. 경쟁이 라는 가치를 효과적으로 전달하기 위한 주제 통합 방안으 로서 교육과정 재구성을 활용하는 것이다.

주목할 점은 이상의 선행연구들이 교육과정 재구성이 라는 개념을 각기 다르게 이해하고 있다는 것이다. 본 연 구의 필요성은 이 지점에서 비롯되었다. 실제로는 교육과 정 재구성이라는 개념이 상당히 관념적이고 추상적인 수 준에 머물러 있을 가능성이 크다는 것이다. 실제 학생을 가르치는 학교 현장에 적합하도록 교육과정을 적절하게 재구성하여 활용하라는 권고는 너무나도 당연한 것처럼 받아들여진다. 그러나 곰곰이 한 번 생각해보자. 우리는 (체육)교육과정 재구성이라는 개념을 지나치게 직관적인 수준으로만 이해하고 있는 것은 아닌가? 두루뭉술하게 국가수준 체육과 교육과정에서 제시하고 있는 내용을 조

1) 본 연구의 목적이 체육과 교육과정 재구성의 개념을 (재)탐색 하는 데 있는 점을 고려하여 '교육과정 개발자'(김원정, 유정 애, 2010), '체육 수업 내용 재구성 방식'(신기철, 2007) 등 과 같이 다른 개념을 차용하고 있는 선행연구를 제외하였다. 
금 바꿀 수 있는 것 정도로 이해하고 있지는 않은가? "법 령에 의해 국가수준 교육과정이 고시되는 데 이것을 교육 현장에서 바꿔도 되는 것인가', '바꿀 수 있다면 무엇을 바 꿀 수 있는가', '재구성의 수준과 범위는 어디까지인가'와 같은 질문이 뒤따를 수 있다.

이에 본 연구의 목적은 체육과 교육과정 재구성의 개 념을 탐색하고, 이를 바탕으로 적절한 재구성 실천을 지 원할 수 있는 방안을 모색하는 데 있다. 단, 본 연구에서 는 재구성 실천 지원 방안을 2015 개정 체육과 교육과정 문서에 대한 개선의 차원에서 살펴보고자 한다. 이를 위 해 먼저 지금까지 진행된 세 가지 교육과정 재구성 담론 을 Snyder et al. (1992)와 Seo (2011)의 주장을 중심 으로 분석한다(2장). 선행연구 및 2015 개정 체육과 교 육과정 문서에서 제시하고 있는 재구성의 개념을 분석함 으로써 체육과 교육과정 재구성 설정 방안에 대해 논의한 다(3장). 또한 2015 개정 체육과 교육과정 문서에서 제 시하고 있는 교육과정 재구성 관련 내용을 분석한다(4 장). 마지막으로 교사의 체육과 교육과정 재구성에 대한 이해 및 실천을 지원할 수 있는 교육과정 문서 개선 방안 에 대해 제안한다(5장).

\section{교육과정 재구성 담론 분석}

이 장에서는 교육과정 재구성 관련 연구에서 제시된 교육과정 재구성 개념에 대한 담론을 분석한다. 미리 밝 혀둘 점은 본 연구에서 살펴보는 교육과정 재구성은 그 의미가 교과서 재구성과 다르다는 것이다. Lee et al. (2015)의 연구에 따르면 적지 않은 교육과정 재구성 및 실행 관련 선행연구에서 교육과정 재구성과 교과서 재구 성을 혼용하고 있다. 국가수준 교육과정을 바탕으로 편찬 된 국정 또는 검정 교과서의 내용을 재구성하는 것을 교 육과정 재구성으로 이해하는 것이다(Seo, 2009). 이와 같은 관점에서는 교과서의 전개 순서를 교육과정과 다르 게 변경하거나 혹은 일부 내용을 추가하는 것이 재구성의 대표적인 방법이다(Kim, 2004, 2005).

본 연구의 목적 중의 하나는 체육교사가 국가수준 체 육과 교육과정을 이해-재구성-실천하는 일련의 과정을 통해 재구성을 개념적으로 살펴보는 것이다. 단위학교에
서 실천되는 체육 수업이 국가수준 체육과 교육과정의 내 용 즉, 내용체계표와 성취기준을 근거로 설계된다는 점과 교과서는 교육과정을 실제 수업으로 구현할 수 있는 여러 가지 예시(활동, 구체적 수업 방법 등)들을 제공하는 별 도의 자료인 점을 고려하면 교육과정 재구성과 교과서 재 구성은 엄연히 다른 개념이라고 할 수 있다.

교육과정 재구성 담론 분석을 위해서는 선행연구에서 제시한 내용을 종합하는 것이 필수적이다. 본 연구에서는 '교육과정 재구성', '교육과정 적용', '교육과정 해석', 'curriculum reconstruction', 'curriculum redesign', 'curriculum implementation' 등과 같은 키워드를 입력 하여 국내외에서 수행된 교육과정 재구성 관련 선행연구 를 수집하였다. 이중에서 교육과정 재구성을 '국가수준 교육과정을 단위학교 여건에 맞게 변형하는 것'으로 다소 직관적인 방식으로 이해한 채로 관련 현상(예: 초임 체육 교사가 겪는 사고 변화)를 탐색하는 연구(예: Zae \& Yoon, 2015)는 담론 분석 대상에서 제외하였다. 최종적 으로 '재구성'과 '교육과정 재구성' 담론 분석을 위해 본 연 구에서 활용된 선행연구는 8편이다(Hur, 2011; Kim, 2015; Lee et al., 2015; Seo, 2009, 2016; Seo, 2011; Snyder et al., 2011; Sung \& Lee, 2009).

교육과정 재구성 담론 분석을 위해 본 연구에서 활용 한 전형적인 분석틀(analytical framework)은 없다. 이 는 교육과정 문서에서 재구성을 의미하는 내용을 분석하 는 연구가 진행되지 않은 학술적 여건에 기인한 것이다. 교육과정 문서를 분석하고 평가하기 위한 모형 $(\mathrm{BEKA}$ model)을 제안하는 연구가 수행되기도 했으나(Hall, 2013), 연구진은 이 모형을 본 연구와 같이 분석해야 할 개념(교육과정 재구성)이 뚜렷한 경우에 활용하는 것이 적절하지 않다고 판단하였다. 이에 본 연구에서는 지금까 지 진행된 교육과정 재구성 담론을 종합하고 재구성에 대 한 다양한 개념을 주택개조에 비유하는 방식으로 논의를 전개한다(아래 참고).

교육과정 재구성이라는 개념은 '교육과정'과 '재구성'이 라는 두 가지 세부 개념으로 해체될 수 있다. 본 연구의 목적과 우리나라가 국가수준 교육과정을 고시하고 있는 맥락을 고려하면 첫 번째로 제시되는 교육과정은 '국가수 준 교육과정 문서에서 제시하는 내용'으로 이해할 수 있 다. 체육과를 예로 들면, 2015 개정 체육과 교육과정 문 
서에서 제시하고 있는 체육의 성격 및 역할, 가르쳐야 할 내용(내용체계표 및 성취기준), 교수·학습 방법, 평가 영 역에서 제시하고 있는 가이드라인이라고 할 수 있다. 이 와 같은 측면에서 교육과정 개념을 추가적으로 논의하는 일은 필요하지 않다.

문제는 재구성이다. 재구성(再構成, re-design, re-construction)2)이라는 의미를 이해하기 위해서는 '다 시(再)'에 주목해야 한다. 재구성은 원형 (prototype)을 ‘바탕으로' 무엇인가를 '한 번 더 만들어낸다’는 의미를 내 포하고 있다. 원형에서 제시하고 있는 핵심 내용이나 구 조에서 완전히 벗어나는 것을 의미하지 않는다. 이와 같 은 측면에서 교육과정 재구성은 '편곡(re-make)'과 유사 한 개념으로 이해될 수 있다(Seo, 2011). 우리나라 맥락 에서의 교육과정 재구성을 다소 직관적인 방식으로 정의 하면, 교사들이 단위 학교 상황(교육 환경) 및 학생의 능 력 및 요구(학습자 특성) 등을 고려하여 국가수준 교육과 정에 일정 부분 변형을 가하는 것이라고 할 수 있다. 교사 에게 교육 실천과 관련하여 일정 수준의 자율권을 보장하 는 개념으로 해석될 수 있다. 동시에, 국가수준 교육과정 이라는 틀 안에서만 재구성 할 수 있기 때문에 교사의 교 육과정 재구성은 결정권의 범위가 제한적임을 암시하는 개념이기도 하다( $\mathrm{SeO}, 2009)$. 이와 같은 측면에서, $\operatorname{Kim}(2015)$ 은 교사의 교육과정 재구성은 국가-지역-단 위학교 수준에서 제시하는 교육과정을 바탕으로 만들어 질 수밖에 없는 구조이기 때문에 종속 관계에 그칠 수밖 에 없다고 주장한다.

이상은 포괄적인 의미의 교육과정 재구성 개념이다. 문제는 이러한 개념적 정의 방식을 사용하면 실제 교육과 정 재구성과 관련된 구체적 이해와 논의를 진전시킬 수 없다는 것이다. 즉, '학생의 능력 및 요구를 고려한다는 것은 구체적으로 무엇을 의미하는가', '다시 구성한다는 것은 어떤 수준까지의 가능한 것인가' 등과 같은 추가적

2) 구글 학술 검색엔진(google scholar)에서 'curriculum reconstruction'이라는 키워드로 논문을 검색하면 Jansen (1989)과 Briggs (1923) 의 두 편의 연구만 확인할 수 있 다. 두 연구가 발표된 시점이 지나치게 오래된 점을 고려하면 reconstruction은 널리 활용되고 있지 않다고 할 수 있다. 반 면 redesign이라는 용어는 널리 활용되고 있다. curriculum redesign 으로 검색을 하면 2018년 1월부터 발표된 연구가 12 편이 넘을 정도로 적극적으로 수행되고 있음을 알 수 있다.
인 질문이 뒤따를 수 있다. 이에 '개념적으로 그려내는 내 포와 외연도 범위도 명확하지 않다. 이것을 한마디로 응 축시키면 교육과정 재구성은 개념적으로 매우 모호한 무 엇이다' $(\mathrm{Seo}, 2011$, p. 82)라는 다소 과격한 주장이 제기 되기도 하였다. 본 장에서는 Snyder et al. (1992)이 제 기한 세 가지 교육과정 실행관점과 $\mathrm{SeO}$ (2011)의 교육과 정을 이해하는 두 가지 접근을 바탕으로 지금까지 제기된 교육과정 재구성 분류 방식을 분석한다.

\section{금과옥조로서의 교육과정: 원형의 강조}

Snyder et al. (1992)은 교사가 교육과정을 이해하고 이행하는 대표적인 방식 중의 하나로 충실도(fidelity)를 제시하였다. 충실도는 교사가 (국가수준) 교육과정의 내 용을 온전하게 이해하고 이를 '정확히' 전달하기 위한 노 력(의무)으로 이해할 수 있다. 이 관점에서는 교육과정이 란 해당 교과 교육과정 전문가가 개발한 문서라는 것이 전제된다. 교육과정을 개발하는 전문가와 해당 교육과정 을 단위학교에서 실천하는 교사는 이질적인 장소에 위치 하고 있다. 이 관점은 교사가 전문가들이 개발한 교육과 정의 철학, 교육 내용, 교수.학습 방법, 평가가 해당 학교 에서 얼마나 충실하게 적용되는 지에 관심이 있다.

$\operatorname{Seo}(2011, p .82)$ 는 국가수준 교육과정을 '완성된 하 나의 실체'로 보는 것을 실체관적 접근이라고 명명했다. 주목할 점은 완성된 하나의 실체라는 표현을 사용했다는 것이다. 일종의 금과옥조인 셈이다. 이는 국가수준 교육 과정이 법령에 의해 고시되고 각 교과 교육과정에서 제시 하는 성취기준을 반드시 준수해야 하는 우리나라 교육과 정 상황과 유사하다. 개발자와 사용자가 다르다는 점에서 실체관은 외부에서 제공되는 교육과정으로 이해되기도 한다.

충실도 관점이나 실체관적 접근에서는 교육과정 원형 을 최대한 훼손 없이 잘 살려서 그대로 교실까지 전달하 는 것이 중요하다. 이 관점에서는 교육과정 재구성이 강 조되기 어렵다. 예를 들면, 국가에서 개발한 2015 개정 체육과 교육과정이 시·도 교육청-교육지원청-단위학교교사로 전달되는 과정에서 재구성이 자주 일어날수록 구 현되는 최종 결과물(체육 수업 등)은 원형 (2015 개정 체 육과 교육과정)에서 멀어질 가능성이 높다. 편곡을 하면 
할수록 원곡의 특성이 옅어지는 원리와 같다. 이에 충실 도 관점 및 실체관적 접근에서는 교사의 교육과정 재구성 에 대한 범위나 권한은 축소될 수밖에 없다.

물론 축소가 '전혀 없음'을 뜻하는 것은 아니다. 금과옥 조로서의 교육과정이 $100 \%$ 의 충실도로 원형을 추구해 야하는 것을 의미하지는 않는다는 것이다. 교육과정 문서 에서 제공하는 것을 하나도 빠짐없이 실행한다는 것은 현 실적으로 어렵다. 제6차 교육과정에서 일정 수준 이상의 자율성 부여가 제시된 점 역시 이를 뒷받침한다. 결론적 으로, 금과옥조로서의 교육과정에서도 원형 (국가수준 교 육고정)의 형태와 역할에 따라 재구성의 가능성이나 범 위가 달라질 수 있다. 원형에서 구체적인 수준으로 교육 과정 적용 및 실행 관련 가이드라인을 제시하면 재구성의 여지는 매우 적어질 수밖에 없다. 반대로 원형에서 포괄 적인 수준의 가이드라인을 제시한다면 수업을 설계하는 과정에서 일정 수준 이상의 선택권이 제공될 수 있다.

이를 주택 개조에 비유한다면, 원형을 강조하는 충실 도나 실체관적 접근에서는 '인테리어' 수준의 재구성이 가 능하다. 인테리어는 주택의 기본 빼대를 훼손하지 않는 범위 내에서의 추가적인 장식이나 보수를 뜻한다. 교사는 학생의 요구나 학교의 특성 등을 반영해 교수.학습 방법 또는 수업 환경을 바꾸거나(집의 분위기를 바꾸기 위해 벽지를 교체함), 해당 단원의 목표를 달성하기 위해 차시 배분이나 순서 바꾸기 등(아이들의 독서습관을 위해 거 실을 서재처럼 꾸밈)의 재구성(인테리어)을 하는 것이 다. 인테리어로 집을 세련되게 꾸밀 수 있는 것처럼 교육 과정 재구성을 통해 더 높은 수업 효과를 기대할 수 있다.

물론, 위에서 언급한 인테리어 변화 수준(교육과정 문 서에서 제시하고 있는 '적용'과 관련된 가이드라인 준수)을 교육과정 재구성으로 개념화하는 방식의 적절성에 대해서 는 논의의 여지가 있다(3장의 2절에서 구체적으로 논의). 다만, 현재까지 논의되고 있는 교육과정 재구성에 대한 담 론과 제 6 차 교육과정에서 처음으로 제시된 교사의 교육과 정 해석 및 적용 관련 자율성을 고려하면 인테리어 변화 수 준 역시 재구성의 한 방식으로 보는 것이 적절하다.

\section{교육현장에서 만드는 교육과정: 새로운 원형 구성}

Snyder et al. (1992)의 충실도 관점과 $\mathrm{Seo}(2011)$ 의
실체관적 접근에서의 국가수준 교육과정은 학교 밖에서 제공되는 이른바 '주어지는 교육과정'이라고 할 수 있다. 흥미로운 점은 두 연구 모두에서 반대의 개념도 함께 제 시하고 있다는 것이다.

Snyder et al. (1992)은 실제 수업이 실천되는 현장에 서 교사와 학생들이 함께 만들어가는 교육과정을 생성 (enactment) 관점으로 정의한다. 외부에서 주어지는 교 육과정이 아니라 해당 현장에서 가치 있다고 생각하는 자 체 교육과정을 개발한다는 점에서 앞서 제기한 충실도나 실체관적인 접근 관점과 대비되는 것이다. 생성의 관점에 서는 생성의 주체가 되는 교사의 교육철학이나 각 교사가 교육과정을 인식하는 틀이라고 할 수 있는 '교육과정 관'(Lee et al., 2015, p. 81)이 매우 중요할 수밖에 없다. 즉, 생성의 관점에서의 교육과정 재구성은 '내가 추구하고 자 하는 교육의 목적은 무엇인가', '현재 학생들이 반드시 함양해야 할 역량은 무엇인가', '이를 위해서 필요한 교육 내용 및 방법은 무엇인가' 등을 바탕으로 교사가 주체적으 로 이상적인 교육을 설정하고 이를 실천하는 데 가장 효과 적인 교육과정을 개발하고 적용하는 것을 의미한다.

$\operatorname{Seo}(2011$, p. 82-83)가 제시하는 '텍스트관'은 국가수 준 교육과정을 수업을 설계하고 실천하는 데 활용할 수 있는 또 다른 문서 중의 하나로 이해하는 것이다. 텍스트 관에서의 국가수준 교육과정은 학교체육정책, 교과서, 교수·학습 자료, 시설 및 용·기구 등과 같이 교육과정 계 획이나 수업 설계 및 실천에 영향을 줄 수 있는 여타의 요 소들과 동등한 지위를 가지는 것이다. 언급한 다양한 요 소들은 교사와 학생이 추구하는 체육교육이나 바람직한 체육 수업에 기여하는 수단 또는 도구로 활용되는 것들이 다. 여기에 국가에서 제공하는 교육과정 역시 교사와 학 생이 추구하는 체육교육이나 체육 수업에 기여하기 위해 활용되는 것이며, 추구하는 방향과 다른 교육과정이라면 선택되지 않을 수도 있다는 것이 텍스트관의 관점이다. 이와 같은 측면에서 텍스트관은 교사와 학생이 함께 만들 어가는 교육과정이라고 할 수 있다.

생성 접근 또는 텍스트관 관점은 앞에서 언급한 충실 도나 실체관적 접근과는 국가수준 교육과정의 역할에 대 해 완전히 다르게 인식하고 있다. 이 관점에서는 국가교 육과정이 학교의 체육교육이나 수업을 위한 예시 자료 또 는 참고 자료에 불과하다. 
주택 개조로 비유하자면, 생성 또는 텍스트관에서의 재구성은 '재건축'에 해당한다고 볼 수 있다. 재건축은 현 재 건물의 상태가 사용하기에 적절하지 않은데 약간의 변 형으로는 그 용도나 목적을 충족할 수 없기 때문에 건물 (주택)을 부수고 새로 짓는 방식이다. 즉, 생성이나 텍스 트관은 필요에 따라서는 재건축과 같은 수준까지의 교육 과정 재구성이 필요하다는 것을 전제로 하는 관점이다.

시공하는 사람의 전문성 역시 인테리어와 재건축을 구 분하는 중요한 요소 중의 하나이다. 인테리어는 집 꾸미 기에 관심이 있거나 손재주가 있는 사람이라면 어렵지 않 게 시도할 수 있다. 재건축은 다르다. 공인된 자격과 전문 성을 갖추어야 시행이 가능하다. 그 일이 간단하지 않기 때문에 아무나 시행할 수 없다. 생성 또는 텍스트관에서 의 재구성을 위해서는 교사의 교육과정 전문성이 무엇보 다 중요하다고 할 수 있다. 명확한 체육교육 철학을 가지 고 목표를 달성하는 데 가장 효과적인 수업 내용, 방법, 평가를 포함한 교육과정을 개발해야 하기 때문이다.

교육의 최종 수요자인 학생과 함께 교육과정을 만들어 간다는 것은 이상적인 모습으로 이해될 수 있다. 학습 효 과를 극대화하기 위해서 구성주의 관점을 적극적으로 적 용하고 학습자 중심의 수업을 설계하는 것은 앞으로도 추 구해야 할 목표 중의 하나다. 문제는 생성 접근 또는 텍스 트관 관점을 우리나라에서 온전하게 적용할 수 있는가이 다. Lee et al. (2015)에 따르면 법령에 의해 국가수준 교육과정이 고시되는 우리나라에서는 생성 관점 자체가 성립될 수 없다는 주장도 제기된다. 개정·고시되는 교육 과정이 국가-지역-학교-교사로 이어지는 하달의 흐름을 갖는다는 점에서 그렇다는 것이다. 실제로 시·도 교육청 과 교육지원청 수준에서 제시하는 교육과정 적용에 대한 지침은 국가수준 교육과정과 거의 같은 형태로 제시되고 있다(Lee et al., 2017). 교사 입장에서는 상위 기관에서 제시한 가이드라인을 반드시 준수해야 한다. 국가수준 교 육과정이 단위학교 교육과정 편성 및 교과서 개발 등과 같은 실제 체육교육 실천에 상당한 영향력을 갖는다는 점 을 고려하면 생성 및 텍스트관은 우리나라에서 실제로 실 천되기 어렵다고 볼 수 있다.

\section{부분 재구성의 교육과정: 원형과 재구성의 역할 구분}

이상에서 살펴본 두 가지 접근인 '금과옥조로서의 교육 과정'과 '교육현장에서 만드는 교육과정'은 국가수준 교육 과정을 바라보는 관점에서 서로 대비된다. 한 쪽에서는 국가수준 교육과정에서 추구하는 철학을 정확하게 이해 한 후 이를 충실하게 준수해야 하고, 다른 한 쪽에서는 국 가수준 교육과정은 참고할 뿐 개별 교사의 교육철학이나 교육과정에 대한 관점이 가장 중요하다. 교육과정을 개발 하고 적용하는 주체나 기준에 대한 스펙트럼이 있다면 이 들은 양 극단에 위치하는 것이다.

이 두 가지 접근의 중간 지점에 Snyder et al. (1992) 가 제시한 상호조정(mutual adaptation) 관점이 있다. 이 관점은 외부에서 제공되는 교육과정의 기준이 있고 이 것을 학교 현장에서 적용하는 것에 관심이 있다. '관심이 있다'는 것은 제공되는 교육과정을 완벽하게 이행하는 것 이 쉽지 않은 일이며, 완벽한 이행만이 교육의 성공을 담 보하는 것이 아니라는 점을 전제한다(Seo, 2016). 상호 조정 관점을 우리나라 체육교육 맥락으로 적용한다면, 2015 개정 체육과 교육과정을 $\mathrm{A}$ 학교에 적용하는 데 어 떤 어려움이 있는가', '이 어려움을 해결하기 위해서 B교 사가 실천하고 있는 방법은 무엇인가', '안전 영역을 실천 하기 위해서 C와 같은 방법을 활용한 이유는 무엇인가' 등과 같은 질문을 하고 각 질문에 대한 답을 찾는 과정들 을 재구성으로 볼 수 있다.

'상호'와 '조정'이라는 두 가지 용어가 제시하는 것처럼 상호조정의 관점은 서로 다른 기관(입장)들이 교육과정 을 조정해 가는 이상적인 관점으로 이해될 수 있다. 그러 나 우리나라 교육과정에서 국가수준 - 시·도 교육청 - 교 육 지원청 - 단위 학교 - 교사의 연결 고리 과정은 보다 선 형에 가깝다. 앞에서부터 전달된 것을 바탕으로 다음의 것이 만들어지기 때문에 양방향의 조정이라는 관점으로 설명하는 것이 적절하지 않을 수 있다. 그럼에도 국가수 준 교육과정이라는 원형을 중심 다른 기관의 입장이 덧대 어지는 형태가 보장되어 있으며, 그 결과로 개선된 교육 과정이 개발된다는 점을 고려하면 일종의 상호조정으로 이해할 수도 있다.

이에 상호조정은 교사가 국가수준 교육과정을 비판 없 이 수용하는 것을 지양하고, 가르치는 환경을 둘러싸고 
있는 다양한 맥락적 요소를 고려하여 일종의 변형을 가하 는 것이라고 할 수 있다. 국가수준 교육과정을 이해하고 이를 바탕으로 단위학교 교육과정을 개발하는 과정에서 현장 적용 가능성 및 교육 효과를 높이기 위해 일종의 수 정, 변형, 보완 등의 재구성을 하는 것이다. 그렇기 때문 에 상호조정의 관점에서는 국가수준 교육과정이 추구하 고자 하는 내용의 현장 적용 가능성을 비판적으로 살펴보 는 노력이 요구된다.

우리나라와 같이 국가수준 교육과정이 지침서 성격의 문서로 제공되는 점과 교육과정 개발의 주체(교육부 및 각 교과교육과정 개발팀)와 실행자(초·중등교사)가 명확 히 구분되는 측면을 고려하면 상호조정 관점 역시 충실도 접근과 많은 부분을 공유한다고 할 수 있다(Lee et al., 2015; Seo, 2016). Snyder et al.(1992) 역시 상호조정 관점은 충실과 생성 사이에 위치하며 그 특징이 명확하게 구분되지 않는다고 언급하고 있다. 본 연구에서는, 충실 도 및 실체관적 접근에서 논의한 바와 같이, 원형에서 제 시하는 교육과정 적용과 관련된 가이드라인의 구체성 정 도에 따라 충실도와 상호조정의 두 관점을 구분하고자 한 다. 원형을 준수해야 할 그 범위가 크고 실제 적용 과정에 서 학교나 교사들이 재구성 할 여지가 거의 없도록 가이 드라인이 제시된다면 충실도 접근에 좀 더 가까울 것이 다. 그러나 2015 개정 체육과 교육과정과 같이 대강(大 綱, slimming 또는 simplification)화된 문서 수준의 원 형이라면 교육 환경의 다양한 특성을 고려해 적용하기 위 한 크고 작은 재구성이 반드시 필요하기에 상호조정 관점 으로 이해할 수 있다.

다른 관점과 마찬가지로 주택 개조에 비유하면, 상호 조정 관점은 '리모델링'과 흡사하다. 리모델링은 기본적 인 주택(건물)의 틀을 크게 손상시키지 않으면서 구조를 변경하는 것이다. 두 개의 방을 합하여 하나의 큰 공간을 만들거나 욕조를 없애고 샤워부스로 바꾸는 등의 인테리 어 수준의 변화를 넘어서는 형태의 개조이다. 그렇다고 재건축처럼 원래의 집을 완전히 없애고 새로운 형태의 집 을 만드는 형태는 아니다.

주목해야 하는 점은 리모델링은 내력벽과 같이 절대로 건드려서는(재구성해서는) 안 되는 건축물의 구조가 있 음을 전제한 개조 방식이라는 것이다. 침실, 화장실, 주방 등 주택에 반드시 필요한 공간들은 그 형태가 바뀌더라도
기능은 반드시 남아있어야 한다는 점 역시 중요하다. 상 호조정의 관점에 적용하면, 교사는 교육과정의 내력벽에 해당하는 것이 무엇인지 파악하고 이는 원형 그대로 유지 해야 하며, 교육과정을 재구성하는 과정에서도 목적 및 기능이 반드시 유지되어야 하는 부분을 인지하고 있어야 한다.

\section{체육과 교육과정 재구성 의미 탐색}

본 장에서는 선행연구와 2015 개정 체육과 교육과정에 서 제시하고 있는 체육과 교육과정 재구성의 개념과 의미 에 대해 분석하고 이를 바탕으로 체육과 교육과정 재구성 개념의 설정과 이해에 나타나는 쟁점에 대해 살펴본다.

\section{선행연구에서의 체육과 교육과정 재구성}

그동안 수행된 체육과 교육과정 재구성 관련 연구들은 많지 않다(Kim \& Lee, 1999; Chang \& Ko, 2006; Park \& Park, 2006; Kim et al., 2012; Kim \& Lee, 2013; Zae \& Youn, 2017). 선행연구에서 체육과 교육 과정 재구성의 개념에 대해 이해하는 관점은 다음의 세 가지로 요약된다3). 첫째, 교육과정에서 제시하고 있는 내용(요소) 구성을 자신의 수업에서 가르칠 내용(신체활 동)으로 구체화하여 수업을 설계하는 것이다. 예를 들면, Kim \& Lee (2013)는 고등학교 전문교과 체육계열 교육 과정 중 '체육과 진로탐구' 교과에서 제시하고 있는 내용 요소가 '스포츠 개론' 또는 '스포츠경기과학'의 내용 요소 와 중복되는 점을 지적하고, 체육고등학교 학생들의 특수 성을 고려한 프로그램을 개발하는 것을 체육과 교육과정 재구성으로 개념화하였다. Park \& Park (2006)의 연구 에서도 초등학교 교사들은 학생의 흥미도 및 지도 역량 등을 이유로 표현·체조활동보다 게임·육상 활동에 초점 을 맞추어 재구성한다고 보고하고 있다.

둘째, 수업 목표를 교육과정에서 제시하는 것과 다르

3) 서론에서 제시한 바와 같이 대부분의 연구는 뚜렷한 체육과 교육과정 재구성 개념을 제시하지 않은 채로 수행되었다. '체 육과 교육과정 재구성은 이와 같은 개념이다'라고 제시한 연 구는 없다. 
게 설정하고, 새로운 목표를 달성하기 위한 수업 내용을 구성하는 방식의 재구성이다. Chang \& Ko (2006)은 2007 개정 체육과 교육과정에서 강조하는 '신체활동 가 치'의 내면화가 그동안 체육과가 '기능교과'라고 비판받은 점을 극복할 수 있는 방안인 점에 주목하였다. 이에 초등 학생들에게 '건강'이라는 신체활동 가치를 효과적으로 지 도하기 위해 제 7 차 체육과 교육과정의 6 학년 내용 요소 중에서 경쟁적 요소를 추출하여 교육 프로그램을 개발하 였다. 이와 같은 방식은 내용 요소를 (재)조합 한다는 측 면에서는 첫 번째 방식과 공통점이 있지만, '운동기능 숙 달'에서 '신체활동 가치 내면화'라는 목표 수준의 변화가 동반된다는 점에서 차이가 있다고 할 수 있다.

셋째, 재구성을 교육과정을 해석하고 이를 바탕으로 체육 수업을 설계 및 실천하는 일체의 과정이라는 다소 폭 넓은 개념으로 설정하는 것이다. 이는 국가수준 체육 과 교육과정을 주체적으로 이해하여 '교사 수준의 교육과 정 개발하기'로 이해될 수 있다(Zae \& Youn, 2015, p. 261). 이와 같은 방식은 Kim et al. (2012)에서 명확하 게 제시된다.

체육교육과정 재구성 계획 경험은 '체육교사의 교육철학', '학교의 시설 및 용구', '체육교사들 간 협의', '학생 및 학 급', '운동 종목 및 기능', '수업 환경'으로 나타났다. 그리고 체육교육과정 재구성 실천 경험은 '목표는 내가 추구하는 체 육 수업', '내용은 다양한 스포츠 경험을 통한 신체 활동 가 치의 의미 전달', '방법은 대안을 찾기 위한 방법 적용', '평 가는 또 하나의 유익한 수업 과정'으로 분석되었다.(p. 31)

종합하면, 선행연구에서는 재구성에 대해 작게는 '교 육과정의 내용 요소를 바탕으로 학습 내용을 다시 구성하 는 것' 또는 '목표를 수정해 그 목표 달성을 위해 내용 요 소를 다시 구성 하거나 조정하는 것’으로, 크게는 '국가수 준 교육과정 문서를 해석해 새롭게 교사수준 교육과정을 개발하는 것'이라고 설정하고 있다. 중요한 점은 어떤 경 우에도 재구성의 기준이나 재구성을 해서는 안 되는 영역 또는 재구성이 가능한 범위 또는 수준에 대한 생각들을 뚜렷이 제시하지 않았다는 것이다.

\section{5 개정 체육과 교육과정 문서에서의 재구성 의미 탐색}

2015 개정 체육과 교육과정 문서에서 직접적으로 재
구성을 언급하거나 재구성과 관련된 내용으로 해석될 수 있는 내용은 많지 않다. 따라서 단위학교에서는 연간 교 육과정을 개발하는 과정에서 재구성 가능 여부나 범위에 대해 자의적으로 해석할 여지가 있다. 이는 교육과정에서 추구하고 의도하는 방향과 다른 수업 설계로 이어질 수 있다는 측면에서 바람직하지 않다. 본 절에서는 2015 개 정 체육과 교육과정의 '성격 및 목표', '내용 체계(표)', '성 취 기준', '교수·학습 및 평가의 방향' 항에서 제시하고 있 는 재구성과 관련된 내용을 분석한다.

\section{성격 및 목표}

성격 항이나 목표 항에서는 재구성과 직접적으로 관련 되는 내용은 없다. 성격 항에서는 체육 교과 역량 함양이 기존 교육과정과 구분되는 결정적인 차이점이라는 것과 체육과의 본질 및 역할을 중심으로 진술하고 있다. 체육 교육을 통해 추구해야 하는 가치를 다소 추상적인 형태로 기술하고 있기 때문에 교사가 재구성 할 수 있는 내용이 라기보다 이해를 통해 교육 및 수업의 방향으로 삼아야 하는 영역으로 이해하는 것이 적절하다. 목표 항 역시 다 섯 가지 영역과 학생들의 함양해야 할 체육과 역량과의 연결고리를 제시하고 있는 점을 고려하면 재구성과 관련 된 내용은 없다고 할 수 있다. 종합하면, 성격 항과 목표 항에서는 학교에서의 체육교육이 지향해야 할 실천 방향 에 대해 일반적이고 암묵적인 형태로 제시하고 있다.

\section{내용 체계(표)}

내용 체계(표)는 '재구성'과 '적용'의 경계 설정과 관련 된 내용을 포함하고 있다. 앞서 2장에서 논의한 바와 같 이, 재구성은 원형 (구성품)의 존재가 전제되어야 한다. 재(再)구성이 원형에서 제시하고 있는 핵심 내용을 바탕 으로 무엇인가를 다시 조직해내는 것이기 때문이다. 이와 같은 입장에서 체육교사는 재구성에 앞선 일종의 구성품 (package)을 확인할 수 있어야 한다. 그러나 2015 개정 체육과 교육과정에서 제시하고 있는 내용 요소는 완성되 어 있는 일종의 구성품이 아니라, 체육교사가 활용해 만 들어내야(조직해야) 하는 교육 내용을 펼쳐놓은 형태에 가깝다. 중학교 2학년 학생을 대상으로 도전 영역을 가르 
치고자 하는 체육교사를 예로 들면, 먼저 투기 도전 활동 으로서 태권도라는 신체활동을 선정할 수 있을 것이다. 이후 내용 체계표를 근거로 선정한 신체활동인 태권도가 올림픽 종목으로 채택된 과정 (태권도의 역사), 송판 격파 의 충격 에너지(과학적 원리), 여러 가지 발차기(경기 기 능 및 방법), 태권도 훈련 및 경기에서의 덕목(절제)을 수 업에 적용하기 위해 교육과정을 조직할 것이다. 이러한 과정은 재구성의 활동과 유사하면서도 한편으로는 교육 과정에서 제시하고 있는 일종의 가이드라인을 '적용'한 것 으로 볼 수도 있다.

선정할 수 있는 신체활동이 무궁무진하다는 점은 적용 (선정)4)과 재구성의 차이를 더욱 어렵게 만드는 요인이 다. 2007 개정 체육과 교육과정에서 처음으로 도입된 신 체활동 가치 내면화 기조는 현재까지 이어지고 있다. 다 섯 가지 신체활동 가치를 배우는 것이 체육과의 중요한 목적 중의 하나이며, 이를 위해 다섯 가지 신체활동 가치 를 '(내용)영역'에 위치시켰다. 이에 체육교사는 해당 신 체활동 가치를 내면화하는 데 도움이 되는 '적절한' 신체 활동을 선정할 수 있다. 이는 신체활동 자체가 아닌 신체 활동 가치에 대한 학습이기 때문이다. 해당 학년군이나 영역별로 활용할 수 있는 신체활동이 제시되어 있지만 어 디까지나 예시에 불과하다. '학교의 교육 여건을 고려하 여 다른 영역의 신체활동 예시나 새로운 신체활동을 선택 할 수 있다'고 교육과정 문서에 언급한 바와 같이 어떤 신 체활동에 대한 학습도 전혀 강제성을 가지지 않는다 (Ministry of Education, 2015b, p. 19). 이와 같은 관 점에서는 체육교사가 교육을 위해 신체활동을 선정하는 일은 교육 내용의 재구성이 아니라 교육과정에서 제시하 고 있는 가이드라인을 충실히 '적용'하는 것으로 이해할 수 있는 것이다.

이상에서 논의한 내용은 교육 내용의 위계성 및 계열 성과 밀접한 관련을 지닌다. 수학, 사회, 과학 등과 같은 교과는 학생의 발달 수준을 고려한 교육 내용의 위계성 설정이 매우 중요하다. 방정식을 배우고 난 후에 부등식 을 배우는 것과 같은 원리다. 실제로 이상에서 언급한 주 지교과에서는 교육과정이나 교과서에서 제시하는 내용

4) 체육과 특성 상 신체활동 선정에 의해 실제 수업의 양상이 크 게 달라질 수 있는 점을 고려하면 체육과의 교육과정 적용은 신체활동 선정과 깊게 관련될 수밖에 없다.
을 보충하기 위해 일부 교육 내용을 추가·수정·보완하는 것을 교육과정 재구성으로 이해한다(Kim, 2005). 그러 나 교육내용의 계열성에 크게 영향을 받지 않는 체육과에 는 이런 방식의 재구성이 해당되지 않는다. 2015 개정 체 육과 교육과정 체제에서도 학년에 따른 교육내용의 계열 성 구분을 찾아내기 어렵다. 학년군제 하의 중학교에서는 학년에 따른 교육내용의 계열성 자체를 나타내지 않는다. 초등학교의 경우, 3-4학년군과 5-6학년군에서 배워야 할 내용이 구분되어있다. 예를 들면, 속도 및 동작 도전과 거 리 및 표적/투기 도전이 3-4학년군과 5-6학년군에 각각 포함되어 있는 방식이다. 그러나 이와 같은 구분 방식이 학생의 발달수준이나 교육내용의 계열성에 근거했거나 적합하다는 설명은 제시되어 있지 않다.

종합하면, 선행연구에서 보고하고 있는 교육과정 적용 (또는 실행)과 교육과정 재구성 간의 개념 혼란은 2015 개정 체육과 교육과정 내용 체계와 다음에 제시할 성취기 준에서도 동일하게 나타나고 있다. 본 연구에서는 국가수 준 교육과정이라는 일종의 원형을 단위학교에서 '적용'하 는 과정에서 발생하는 교사의 일련의 교육적 조처를 교육 과정 재구성으로 설정하고자 한다(체육과 교육과정 문서 에서의 재구성 개념 및 방향성 설정에 대해서는 다음 장 에서 구체적으로 논의). 만약 신체활동 선정이라는 가이 드라인 준수를 교육과정 '적용'으로 보고자 한다면 - 아래 에서 논의할 - 교수·학습 및 평가 영역에서 제시하고 있 는 체육 수업에 대한 사항 및 교육과정에 포함된 내용의 대부분이 '적용'으로 이해될 수 있기 때문이다. 그렇게 된 다면 적용과 재구성을 나누는 것조차 모호해진다. 이에 아래에서부터는 2 장에서 제시한 부분 재구성의 교육과정 ('리모델링' 표현)을 바탕으로 논의를 전개한다.

\section{성취기준}

내용 체계(표)는 내용 항을 개괄적, 핵심적으로 요약 해 안내하고자 제작된 것이며, 실제로 교육해야(되어야) 할 내용에 대하여 구체적으로 설명하는 것은 성취기준이 다. 교사가 2015 개정 체육과 교육과정을 재구성하고 이 를 바탕으로 수업을 설계할 때는 성취기준 이해가 전제되 어야 한다. 성취기준은 체육 교과에서 가르치고 배워야 할 내용을 국가에서 고시한 것이다. 학생들이 반드시 학 
습해야 할 내용이라는 점을 고려하면 진술의 수준과 범위 를 건드려서는 안 되는 일종의 내력벽에 해당한다고 할 수 있다. 따라서 제시된 진술과 다른 내용을 성취기준으 로 설정하는 것은 적절하지 않다.

성취기준(achievement standard)의 정의는 '교육을 통해 학생들이 성취할 것으로 기대되는 것을 명시한 것' (교육평가용어사전)이다. 학습해야 할 구체적 내용이면 서도 도달점인 것이다( $\mathrm{Seo}, 2016) .2015$ 개정 체육과 교 육과정에 따르면 체육 교과의 성취기준은 학생이 신체활 동이나 스포츠에 다양한 경로로 참여함으로써 건강, 도 전, 경쟁, 표현, 안전이라는 영역에 포함된 내용을 학습하 고, 이를 통해 건강 관리, 신체 수련, 경기 수행, 신체 표 현이라는 네 가지 체육과 역량을 함양하는 모습에 대하여 구체적으로 제시한 내용이라고 할 수 있다. 중학교 영역 형 경쟁의 성취기준을 예로 들면, '영역형 경쟁 스포츠의 경기 방법과 전략을 이해하고 경기에 활용할 수 있으며, 경기 상황에 맞게 전략을 진단하여 창의적으로 적용한다' 와 같이 학습을 통해 학생들이 체육과 역량의 함양 또는 신체활동 가치를 내면화하는 모습이 설명 또는 확인되는 진술을 제시하고 있다. 다만 예시에서 볼 수 있는 것처럼, 성취기준이 다소 추상적이고 포괄적인 수준으로 기술되 어 있기 때문에 실제 교수·학습 현장에 투입, 적용하려면 학습 소재나 수업에서 실시할 신체활동의 선택 등 실제화 및 구체화 과정이 필요하다는 점에서 성취기준의 재구성 이 가능하다.

문제는 위에서 논의한 바와 같이 성취기준은 건축물의 내력벽에 해당하기 때문에 재구성 개념에 대한 추가적인 논의가 필요하다. 실제 2015 개정 체육과 교육과정 문서 에서도 다음과 같이 성취기준을 '반드시' 지도하라고 명확 하게 제시하고 있다.

단위 학교에서는 교육과정에 제시된 내용 영역(건강, 도전, 경쟁, 표현, 안전)에 근거하여 각 영역별 성취기준을 해당 학년군(3-4학년, 5-6학년, 중학교 1-3학년)에서 반드시 지도한다(Ministry of Education, 2015b, p. 44)

본 연구에서는 성취기준을 이해하는 관점에 따라 재구 성의 범위가 달라질 수 있는 점을 추가적으로 논의하고자 한다. 성취기준은 - 성취라는 용어가 내포하고 있듯이 학생들이 체육수업을 통해 얻을 수 있는 일종의 교육적
'결과'로 보는 것이 상식적이다. 그러나 포괄적으로 진술 되어 있는 체육과 성취기준에서는 학생의 성취기준 도달 에 대한 상태를 판단하는 데 어려움이 있고, 도달의 수준 을 학습목표의 어느 수준으로 두느냐에 따라 입장이 달라 질 수 있다. 따라서 성취기준을 결과로 보는 관점에서는, 아래에서 논의하는 '구체화'와 함께, 교육과정 문서에서 제시된 성취기준을 학생들이 도달해야 할 '최저기준'으로 설정하고, 체육교사가 제시한(재구성한) 보다 높은(그리 고 가능하면 구체적으로 제시된) 높은 수준의 성취기준 을 추가적으로 제시하는 형태를 교육과정 재구성으로 볼 수 있을 것이다.

성취기준을 수업에서 다루어야 할 '내용'으로 접근하면 재구성의 범위가 달라진다. 우리나라의 경우 적지 않은 교과에서 성취기준을 학생들이 도달해야 할 일종의 '결과' 가 아닌 수업에서 다루어야 할 '내용'의 관점으로 접근하 고 있다(Seo, 2016). 이와 같은 접근은 2015 개정 체육 과 교육과정에서도 확인된다. '성취기준을 반드시 지도한 다'(p. 44) 라고 명시하고 있는 점이다. 이 관점에서의 성 취기준 재구성은 '수업 또는 단원 설계 과정에서의 성취 기준 구체화'라고 할 수 있다. '구체화'라는 표현을 활용한 것은 제시된 성취기준이 수업에 직접 적용하기에는 일반 적이고 포괄적으로 진술되었기 때문이다. 따라서 체육교 사는 학생들이 배우고 익히게 될 한 개 혹은 여러 개의 구 체화된 진술의 성취기준을 설정하고, 이에 도달하기 위한 신체활동 선정, 교수·학습 방법, 단원 내 차시 배분 등의 실제 수업에서 실천되는 과정을 계획해야 한다. 이 과정 및 결과에서 교육과정 재구성이 필요하다.

종합하면, 성취기준을 '내용'으로 바라보는 관점에서는 성취기준을 구체화하는 형태의 재구성이 가능하며, 성취 기준을 '결과'로 바라보는 관점에서는 체육교사가 2015 개정 체육과 교육과정에서 제시하는 성취기준을 '최소 기 준’으로 설정하는 형태의 재구성이 추가될 수 있다.

\section{교수·학습 및 평가의 방향}

2015 개정 체육과 교육과정 문서에서 ‘재구성'이라는 용어가 직접적으로 제시된 부분은 모두 '교수·학습 및 평 가의 방향' 영역 내에 있으며 내용은 다음과 같다. 첫째, 학습자의 다양한 특성을 고려한 학습 과제의 재구성이다. 
'활동 과제의 유형과 수준은 학습 자료, 과제 수행 시간, 활동 공간의 재구성 등에 기반하여 조절'(Ministry of Education, 2015b, p. 41)이라고 표현된 부분이 대표적 이다. 둘째, 학습 활동의 재구성이다. '성취기준에 보다 쉽게 도달할 수 있도록 영역의 특성과 학습 주제, 학생의 특성 및 가용 자원, 학습 환경을 고려하여 학습 활동을 재 구성한다'(p.46)가 대표적이다. 이는 첫 번째에서 제시된 학습자 특성을 고려한 다양한 학습과제 제시와 매우 유사 한 맥락이다. 셋째, 평가 결과에 대한 재구성이다. 평가 결과의 활용 영역에서 '평가 결과는 학습자와 학부모가 쉽게 이해하도록 구체적으로 재구성하여 안내한다' $(\mathrm{p}$. 49)라고 제시하고 있다. 이는 학생의 성취기준 도달 여부 를 직관적으로 이해할 수 있도록 평가 결과 기술 방식을 정교화하라는 권고사항으로 이해할 수 있다.

첫 번째와 두 번째의 교수·학습 방법과 관련하여 제시 하고 있는 재구성은 '수업 설계'과정에서 고려해야 할 일 반적인 사항을 제시하는 것이라고 이해할 수 있다. 주목 할 점은 '성취기준에 보다 쉽게 도달할 수 있도록'이라는 표현이 함께 제시된 것이다. 이는 성취기준의 재구성에 대한 이전 논의 내용에서와 같이, 성취기준을 구체화하는 재구성에서 활용될 수 있는 다양한 교수.학습과 관련된 가이드라인을 제시한 것이라고 할 수 있다. 또한 이는 일 종의 교수·학습 방법상의 재구성에 초점을 맞춘 진술이라 고 할 수도 있다(Seo, 2009). 실제로 2015 개정 체육과 교육과정에서는 체육과 역량 함양을 지향하는 교수·학 습, 학습자 특성을 고려한 수준별 수업, 자기주도적 교수. 학습 환경 조성, 전인적 발달을 위한 통합적 교수·학습, 맞춤형 교수·학습의 선정과 활용, 정과 외 체육활동과 연 계한 교수·학습의 주요한 교수·학습의 방향에 따라 구체 적인 수업 과정, 내용의 조직 및 배치, 수업 진행 방법 및 전략, 활동 적용(학습)의 방식 등을 설정하기를 권고하고 있다.

문제는 문서에 '방향'이라고 접근하고 있는 것과 같이 교육의 방향이나 목표에 의거하여 보다 효과적인 교육을 하기 위한 지나치게 일반적인 내용으로 구성되어 있다는 것이다. 재구성이라는 개념 자체가 '단위학교의 교육 환 경 및 학습자의 특성이 다르기 때문에 맞춤형의 교사 수 준 교육과정을 재개발 할 수 있다(또는 해야 된다)'라는 요구에 의해 만들어진 점을 고려하면, 교수·학습 및 평가
영역에서 진술하고 있는 방식은 이 필요성을 재차 강조하 는 데 그치고 있다.

\section{교육과정 내 재구성 가능성 관련 진술}

단위학교에서 체육과 교육과정 재구성이 실천되기 위 해서는 재구성의 개념에 대한 공통된 이해와 더불어 체육 교사에게 '무엇을' 그리고 '어떤 수준으로' 재구성 할 수 있 는 권한이 있는지가 명확하게 제시되어야 한다. 서론에서 제시된 교사들의 대화와 같이 학교 여건에 맞게 국가수준 교육과정을 재구성 할 수 있다는 것은 모든 교사가 인지 하고 있지만, 재구성 할 수 있는(혹은 없는) '영역', '범위', '수준' 등에 대해서는 인지하고 있지 않다. 이에 대해서 교 육과정 문서에서도, 또한 어느 지침에서도 제시하고 있지 않기 때문이다. 1 년 동안 건강, 도전, 경쟁, 표현, 안전의 다섯 가지 영역을 반드시 가르쳐야 한다는 매우 기본적인 안내만이 체육교사 직무연수나 단위학교 교육과정 개발 과정에서 등장하고 있다는 것도 같은 맥락이라고 할 수 있다5).

3장에서 살펴본 바와 같이, 2015 개정 체육과 교육과 정에서 재구성이라는 용어를 직접적으로 제시한 내용은 모두 '교수·학습 및 평가의 방향' 영역 내에 있다. 본 연구 에서는 2015 체육과 교육과정 문서에서 재구성 가능성을 살펴볼 수 있는 내용들에 대하여 추가적으로 탐색하였다. 본 장에서는 의무 사안과 허용 사안을 중심으로 2015 개 정 체육과 교육과정 문서에서의 재구성에 대하여 추가적 으로 논의하고자 한다.

의무 사안을 찾아보기 위해 먼저 '반드시’라는 부사를 찾기 기능으로 탐색하였다. 2015 개정 체육과 교육과정 문서에서 '반드시'라는 부사가 표현된 곳은 총 다섯 군데 이다. 모두 '교육과정 운영 계획'에서 제시되고 있으며, '성취기준을 반드시 지도한다'라는 표현으로 문장을 마무 리하고 있다. 성취기준이 국가에서 고시한 체육교육을 통

5) 물론 영역 지도와 관련된 지침은 2015 개정 체육과 교육과정 에 분명하게 제시되어 있다(Ministry of Education, 2015, p. 44). 이와 같은 현상이 지속되는 이유는 적지 않은 체육교 사가 교육과정 문서에서 내용체계표를 제외한 다른 영역을 자 세히 보지 않는 데서 기인하였다고 연구진은 분석하고 있다. 
해 학생들이 성취해야 할 교육적 결과인 점을 고려하면 매우 자연스러운 현상이라고 할 수 있다(성취기준의 재 구성과 관련된 구체적인 논의는 앞에서 다루었음).

다음으로 '해야 한다'라는 종결 어미 역시 일종의 의무 사안으로서의 역할을 나타내는 제시어이다. 흥미로운 점 은 '반드시'라는 부사에서와 같은 엄격한 규제의 내용이라 기보다는, 다음과 같이 '양질의 교육을 위해 체육교사가 노력해야 하는 요소' 정도라는 뜻으로 이해될 수 있는 내 용들이다. '해야 한다'라는 종결어미는 재구성과 직접적 인 관련을 지닌다고 보기 어렵다.

교사는 교수 · 학습 운영을 계획할 때 학생들의 흥미와 수준 을 파악하고 학생들의 다양한 사전 학습 경험을 존중함으로 써 학생에게 유의미한 경험을 제공할 수 있도록 해야 한다. (Ministry of Education, 2015b, pp. 68-69)

허용 사안을 대표할 수 있는 '할 수 있다'라는 종결 어미 는 2015 개정 체육과 교육과정에서 다음과 같이 두 가지 방식으로 활용된다. 첫째, 세부 내용 영역을 지도하는 과 정에서 단위학교의 맥락에 적합하도록 재편성할 수 있다 는 권한 부여이다.

체육과의 학습 내용은 핵년군 단위로 계획하여 구성하며, 건강, 도전, 경쟁, 표현, 안전의 5 개 대영역에서 내용별로 구분된 학 습 영역은 학년별 수준에 따라 단위 학교별로 자율적으로 재편 성 할 수 있다. (Ministry of Education, 2015b, p. 44)

둘째, 다양한 신체활동, 교수·학습 방법, 평가의 활용 을 권장하는 장치로 활용되고 있다. '학생들의 연령별 특 성, 흥미 등을 고려하여 건강을 유지 및 증진하기에 적합 한 놀이, 게임 등을 중심으로 구성할 수 있다'(내용) (p. 12 ), '경기장의 형태와 사용하는 도구, 신체활동에 참여 하는 인원수와 조직의 형태, 실행 규칙 등을 변형하여 활 동을 구성할 수 있다'(교수·학습 방법) (p. 30), '각 내용 요소에 대한 평가 비중을 달리할 수 있다'(평가) (p. 58) 등이 대표적이다. 그러나 이상의 허용사안들은 체육과 수 업을 위한 일반적인 내용을 다루고 있다는 점과 체육교사 의 구체적인 수업 설계에 포함될 수밖에 없는 내용이라는 점을 고려하면 이것을 교육과정 재구성에 대한 언급으로 보기에는 어렵다.

3장과 4장을 종합하면, 2015 개정 체육과 교육과정 문
서에서의 재구성 탐색으로 성격 및 목표 항에서는 재구성 과 관련된 내용을 찾을 수 없었고, 내용 체계(표) 항에서 는 교육과정 '재구성'과 '적용(선정)'의 개념적 경계에 대 한 쟁점이 나타났다. 성취기준에서는 성취기준을 이해하 는 관점에 따라 재구성의 양상이 달라질 수 있음을 확인 하였다. 교수·학습 및 평가의 방향에서는 재구성이라는 용어를 활용하고 있지만, 해당 내용은 체육교사가 수업을 설계하는 과정에서 참고할 일반적인 지침에 가까운 것으 로 분석되었다. 마지막으로 교육과정 재구성 가능 탐색을 위해 추가적으로 제시어를 분석한 결과 성취기준의 재구 성을 나타내는 부사(반드시) 외에 종결어미(해야 한다. 할 수 있다)는 실질적인 재구성과는 관련 없는 것으로 나 타났다.

\section{체육과 교육과정 재구성의 이해와 실천 지원을 위한 제안}

본 장에서는 앞에서 논의된 내용을 바탕으로 체육과 교육과정 재구성의 효과적인 이해 및 실천을 지원할 수 있는 방안을 다음과 같이 두 가지로 제안한다. 이 제안들 은 국가수준 체육과 교육과정 문서의 개선을 토대로 한 것인데, 이는 단위학교에서 교육과정을 개발하거나 체육 교사가 수업을 설계할 때 국가수준 교육과정 문서를 바탕 으로 재구성이 시작된다는 점에 기인한 것이다.

첫째, 재구성의 의미를 명확하게 설정해야 한다. 3장 에서 논의한 바와 같이 선행연구와 체육과 교육과정 문서 에서 '재구성'을 여러 가지 방식으로 이해하고 있었다. '적 용(선정)'과 '재구성'의 경계 역시 분명하게 드러나지 않 고 있다. 또한, 2015 개정 체육과 교육과정의 교수·학습 방법 및 평가에서 재구성으로 제시하고 있는 내용들은 수 업 계획에서 참고할 수 있는 일반적인 가이드라인 제공에 그치고 있다. 이에 (국가수준) 체육과 교육과정을 재구성 한다는 것의 실체가 무엇인지를 체육과 교육과정 문서에 서 명확하게 제시해야 한다.

본 연구에서는 2 장에서 제시한 부분 재구성의 교육과 정('리모델링' 표현)의 수준에서 재구성이 두 가지 다른 층위의 의미를 갖고 제시되기를 제안한다. 첫 번째 방식 
은 구체화 수준의 재구성이다. 내용 체계(표)를 바탕으로 신체활동을 선정하는 것과 교육과정 문서에서 제시된 성 취기준을 실제 교육현장(수업 등)으로 구체화하는 것이 여기에 해당한다. 예를 들면, 국가수준 교육과정의 경쟁 영역에서 반드시 지도해야 하는 내용 요소인 '역사와 특 성', '경기 기능과 과학적 원리', '경기방법과 전략'을 단위 학교의 실천으로 수업에 펼쳐내기 위해서는 하나 또는 여 러 가지의 신체활동을 선정하는 과정이 필수다. 또한 선 정한 신체활동이 가지고 있는 특성에 따라 수업의 양상이 달라지고 학생들이 배우고 익힐 수 있는 성취기준 역시 이에 맞추어 조정될 수밖에 없기 때문에 성취기준의 구체 화라는 재구성이 필연적으로 따라온다. 필연적으로 따라 온다는 뜻은 자발적인 의지가 없더라도 반드시 해야 되는 것이라는 의미이다. 이러한 구체화 수준의 재구성은 소극 적인 형태의 재구성이라고 할 수 있을 것이다.

두 번째 방식은 적극적인 형태의 재구성이다. 3장에서 논의한 바와 같이, 2015 개정 체육과 교육과정의 성격 및 목표 항은 교육과정에서 추구하는 독특한 철학을 담고 있 기 때문에 개별 체육교사의 재구성과 거리가 있고, 교수. 학습 방법 및 평가 항은 효과적인 수업 설계와 관련된 일 반적인 가이드라인을 제시하고 있기에 이 역시 재구성에 벗어나 있다. 결국 2015 개정 체육과 교육과정에서 재구 성과 관련된 항은 내용 체계(표) 및 성취기준이라고 할 수 있다. 내용 체계 및 성취기준과 관련하여 체육교사가 반드시 준수해야 하는 원형 (내력벽)은 '각 영역별 성취기 준을 해당 학년군에서 반드시 지도해야 한다'와 '초등학교 3-4학년군과 5-6학년군에서 가르치는 내용은 다르다' (예: 필드형/네트형 경쟁은 반드시 5-6학년군에서 가르 쳐야 한다) 정도로 볼 수 있다. 본 연구에서는 원형에 대 한 부분 수정이라는 재구성의 입장에서 내용 요소의 학년 군간 이동 및 성취기준의 수정·확장·추가 등의 적극적 재 구성과 관련된 논의가 진전되어야 한다. 이를 위해서는 논리적 근거 확보, 실행 가능성 탐색, 그리고 재구성으로 인해 기대되는 교육적 효과에 대한 기초 연구가 향후 지 속적으로 수행되어야 할 것이다.

둘째, 설정한 재구성의 의미를 체육과 교육과정 문서 에서 분명하게 제시해야 한다. 구체적인 형태로는 각 '항' 에서 제시하는 재구성의 의미가 무엇인지 그리고 어떤 수 준으로 재구성이 가능한지 교사가 이해할 수 있도록 제시
되어야 한다.

선행연구에서 보고된 체육과 교육과정 재구성의 사례 역시 시사하는 바가 크다. Kim \& Lee (2013)는 Wiggins \& McTighe (2005)가 제시한 '거꾸로 교육과 정' 설계 단계(바라는 결과 확인하기, 수용가능한 증거 결 정하기, 학습경험과 수업 계획하기)를 바탕으로 교육과 정을 재구성하였다. 실제로 개발된 프로그램에서는 '동네 헬스장 방문 및 궁금한 점 인터뷰하기' 등과 같은 다양한 활동이 포함되었다. 주목할 점은 연구진이 교육과정 재구 성 단계에서 2007 국가수준 고등학교 전문교과 교육과정 문서와 해설서 및 고등학교를 참고하였으며, 교사 경력이 있는 대학 교수로부터 검토를 받았다는 것이다. 교육과정 재구성을 위해서는 자세한 형태의 해설서 및 전문지식을 갖춘 외부의 지원이 필요한 셈이다. 별도의 해설서 및 외 부의 지원이 없는 상태에서도 체육교사가 재구성의 의미 및 허용 범위를 충분히 인지할 수 있는 방안이 마련되어 야 한다.

첫 번째 방식의 재구성(소극적인 형태)을 성취기준에 제시하는 방안을 예로 들면, 체육교사는 수업을 위해 선 정한 신체활동 또는 스포츠의 특성에 따라 교육과정 성취 기준을 구체화하여 학교 및 교사수준의 성취기준으로 반 드시 재구성해야 합니다.'와 같은 안내문을 포함하는 방 안을 고려할 수 있다. 부사(반드시) 및 종결어미(해야 한 다 또는 할 수 있다)를 활용하여 반드시 재구성해야 하는 (소극적인 형태, 의무) 단계와 재구성 할 수 있는(적극적 인 형태, 허용) 범위 역시 실질적으로 제시되어야 한다. 즉, 차기 체육과 교육과정 문서에서는 어떤 경우에도 준 수해야 하는 영역(원형)이 무엇인지와 재구성이 요구되 는 또한 가능한 영역과 그 범위에 대해 명확하게 제시해 야 한다.

\section{요약 및 제언}

본 연구의 목적은 체육과 교육과정 재구성의 개념을 탐색하고 이를 바탕으로 체육교사의 교육과정 재구성 이 해 및 실천을 지원하는 교육과정 문서 개선 방안을 제시 하는 데 있다. 이를 위해 지금까지 진행된 교육과정 재구 성 담론, 체육과 교육과정 재구성 관련 선행연구, 2015 
개정 체육과 교육과정에서 제시하는 재구성의 의미를 분 석하였다. 본 연구에서는 부분 재구성의 교육과정 ('리모 델링' 표현) 관점을 바탕으로 소극적인 형태와 적극적인 형태의 재구성이라는 두 가지 층위를 도출하였고, 차기 체육과 교육과정 문서 내에 원형과 함께 구별된 재구성의 의미와 범위에 대하여 분명하게 제시할 것을 제안하였다. 후속 연구 수행 관련 제언은 다음과 같다.

첫째, 국외 체육과 교육과정 문서에서 제시하고 있는 재구성의 의미, 범위 및 방법에 대해서 살펴볼 필요가 있 다. 국외 체육과 교육과정은 진술 방식의 상세화(또는 구 체화) 정도에 따라 그 스펙트럼이 매우 다르다. 이를 참고 하여 국외에서 설정하고 있는 체육과 교육과정 재구성의 의미가 무엇이고 향후 우리나라 차기 체육과 교육과정 개 발에 줄 수 있는 시사점이 무엇인지를 탐색할 필요가 있 다.

둘째, 체육과 교육과정 재구성의 교육적 효과에 대해 탐색하는 기초 연구가 필요하다. 직관적인 수준에서 생각 해보면, 원형 추구는 교육의 보편적인 가치를 폭 넓게 보 장한다는 측면에서 장점을 가질 수 있는 반면, 재구성 (변 형)의 강조는 수월성 또는 다양한 환경에서의 교육적 실 효성 제고에 유리할 수 있다. 이러한 관점에서 체육과 교 육과정 재구성의 교육적 효과를 다각도로 살펴볼 수 있는 연구가 수행되길 기대한다.

셋째, 반드시 준수해야 하는 원형 (내력벽) 설정 및 재 구성과의 경계와 관련된 기초 연구가 수행되어야 한다. 교육환경이 급변하고 있다. 교사의 전문성 개발 방식 및 형태 역시 확장되고 있다. 이와 같은 환경에서 원형의 범 위를 어디까지 설정하는 것이 체육교사의 교육과정 재구 성을 촉진할 수 있을 것인지에 대해서도 고민할 필요가 있다. 이를 위해 체육교사가 인식하는 재구성의 필요성 및 희망 허용 범위 등에 대해서 살펴볼 필요가 있다.

\section{참고문헌}

Briggs, T. H. (1923). Curriculum reconstruction in the high school. The School Review, 31(2), 109-115.

Chang, Y. K. \& Ko, S. E. (2008). Reconstruction and application of subject synthesis physical education curriculum in the elementary school. The Journal of Korea Elementary Education, 18(1), 69-95.

Hall, C. (2013). Toward a model of curriculum analysis and evaluatio - BEKA: A case study from Australia. Nurse Education Today, 34, 343-348.

Hur, Y. J. (2011). Importance of teacher's imagination in curriculum reconstruction and implementation. The Journal of Curriculum Studies, 29(1), 137-163.

Jansen, J. (1989). Curriculum reconstruction in post-colonial Africa: A review of the literature. International Journal of Educational Development, 9(3), 219-231.

Jeong, K. S. (2012). Study on the teachers' curriculum literacy. Journal of Curriculum Integration, 6(2), 109-132.

Kim. H. K. (2015). A semantic analysis of the curriculum jaegusung. Journal of Curriculum Integration, 9(2), 54-82.

Kim, K. M. (2014). Prerequisite of curriculum reconstruction. The Journal of Moral Education, 26(1), 195-211.

Kim, A. Y. \& Lee, D. H. (2013). Backwardd designing guidance program in the curriculum of physical education high school. Journal of Learner-Centered Curriculum and Instruction. 13(2), 141-163.

Kim, S. Y. \& Jung, G. S. (2013). Inquiry into the concept of curriculum potential. The Journal of Curriculum Studies, 31(4), 27-50.

Kim, J. P., Park, J. R., \& Park, D. W. (2012). A narrative inquiry on experience of curriculum reconstruction in secondary physical education teachers. Korean Society For The Study of Physical Education, 17(1), 31-50.

Kim, M. E. \& Lee, J. M. (1999). An analysis on reconstruction and operation of physical education curriculum in elementary school. The Korean Journal of the Elementary Physical Education, 5(1), 35-49.

Kim, P. K. (2005). How are secondary teachers reorganizing contents of the textbooks and teachers' guides? The Journal of Curriculum Studies, 23(4), 91-130.

Kim, W. J. \& You, J. A. (2010). Practical dilemmas and strategies of experienced teachers as curriculum makers. Korean Journal of Sport Pedagogy, 17(4), 39-66.

Lee, S. M. (2019). Exploring the directions of the curriculum slimming on the general guidelines of national curriculum : focusing on the needs analysis of teachers and supervisors. The Korean Educational Review, 25(2), 195-224. 
Lee, S. M., Lee. K. E., Yoon, K. J., \& Choi, S. H. (2017). Facilitating the link between local communities and physical and art education at schools. Seoul: Korean Institute for Curriculum and Evaluation.

Lee, Y. M., Jo, S. Y., \& Jung, G. S. (2015). The suggestion for the domestic researches about the perspectives of curriculum implementation. The Journal of Curriculum Studies, 33(3), 79-100.

Lim, Y. (2019). Simplification and specification of the national curriculum documents. The Journal of Research in Education, 32(3), 31-56.

Ministry of Education. (1992). The 6th national curriculum. Seoul: Ministry of Education.

Ministry of Education. (2015a). The 2015 revised curriculum. Sejong: Ministry of Education.

Ministry of Education. (2015b). The 2015 revised physical education curriculum. Sejong: Ministry of Education.

Park, D. G. \& Park, C. B. (2006). The actual conditions of the physical education curriculum reorganization of elementary school teachers. The Korean Journal of Physical Education, 45(4), 307-316.

Park, Y. K., Jeong, S. J., \& Kim, B. S. (2015). A survey of elementary school teachers' perceptions of curriculum and curriculum adaptation. The Journal of Elementary Education, 28(4), 117-143.

Seo, K. H. (2009). Teachers' experience of reconstructing national curriculum. The Journal of Curriculum Studies, 27(3), 159-189.
Seo, K. H. (2016). Curriculum implementation, adaptation, or development?: debate over teachers' role in the curriculum process. The Journal of Curriculum Studies, 34(3), 209-235.

Seo, M. S. (2011). Critique on the conceptual ambiguity of curriculum jaegusung. The Journal of Curriculum Studies, 29(3), 75-91.

Snyder, J., Bolin, F., \& Zumwalt, K. (1992). Curriculum implementation. In P. W. Jackson (Ed.), Handbook of research on curriculum (pp. 402-435). New York: Macmillan.

Sung. Y. K. \& Lee, M. J. (2009). The strategies for the use of content map as a tool of critical curriculum content analysis and its usefulness. The Journal of Curriculum Studies, 27(3), 63-82.

Wiggins, G. \& McTighe, J.(2005). Understanding by design. Alexandria, VA: Association for Supervision and Curriculum Development.

Yoon, K. (2018). Issues needed for the development of the next national physical education curriculum. Korean Journal of Sport Science, 30(2), 360-370.

Zae, S. Z. \& Youn, H. S. (2016). Middle school novice physical education teachers' changes in thinking in the process of curriculum reconstruction for good teaching. Journal of Research in Curriculum \& Instruction, 19(2), 259-280. 


\title{
체육과 교육과정 재구성 개념 및 실천 지원 방향 탐색
}

\author{
윤기준 ${ }^{1}$, 서지영 ${ }^{2}$ \\ ${ }^{1}$ 인천대학교 조교수 \\ 2한국교육과정평가원 선임연구위원
}

[목적〕 본 연구의 목적은 체육과 교육과정 재구성의 개념을 탐색하고 이를 바탕으로 체육교사의 교육과정 재구성 이해 및 실천을 지원하는 교육과정 문서 개선 방안을 제시하는 데 있다. 〔방법〕 교육과정 재구성 담론 관련 선행연구, 체육과 교육과정 재구성 관련 선행연구, 2015 개정 체육과 교육과정에서 제시하는 재구성의 의미를 분석하였다. 〔결과〕 첫째, 교육과정 재구성을 원형 강조, 새로운 원형 구성, 원형과 재구성의 역할 구 분의 세 가지로 구분하였다. 둘째, 선행연구 및 2015 개정 체육과 교육과정에서는 체육과 교육과정 재구성의 의미를 분명하게 제시하지 않고 있다. 셋째, 2015 개정 체육과 교육과정에서는 내용체계 및 성취기준 항에서 재구성이 가능하다. 〔결론) 체육과 교육과정 재구성은 소극적인 의미와 적극적인 의미의 재구성으로 실천될 수 있으며, 향후 체육과 교육과정 문서에서는 재구성의 의미와 허용 범위를 분명하게 제시할 것을 제안하였다.

주요어: 교육과정 재구성, 체육과 교육과정, 2015 개정 체육과 교육과정 